PROCEEDINGS OF THE

AMERICAN MATHEMATICAL SOCIETY

Volume 133, Number 12, Pages 3635-3641

S 0002-9939(05)08181-5

Article electronically published on June 28, 2005

\title{
ON THE LOCATION OF THE DISCRETE SPECTRUM FOR COMPLEX JACOBI MATRICES
}

\author{
I. EGOROVA AND L. GOLINSKII \\ (Communicated by Andreas Seeger)
}

\begin{abstract}
We study spectrum inclusion regions for complex Jacobi matrices that are compact perturbations of the discrete Laplacian. The condition sufficient for the lack of a discrete spectrum for such matrices is given.
\end{abstract}

\section{INTRODUCTION}

Let

$$
J=\left(\begin{array}{ccccc}
b_{1} & c_{1} & & & \\
a_{1} & b_{2} & c_{2} & & \\
& a_{2} & b_{3} & c_{3} & \\
& & \ddots & \ddots & \ddots
\end{array}\right)
$$

be an infinite Jacobi matrix with complex entries. We assume that $a_{n} c_{n} \neq 0$, $n \in \mathbb{N}:=\{1,2, \ldots\}$, and

$$
\lim _{n \rightarrow \infty} a_{n}=\lim _{n \rightarrow \infty} c_{n}=1, \quad \lim _{n \rightarrow \infty} b_{n}=0 ;
$$

that is, the operator $J$ generated by matrix $(\mathbb{1})$ in $\ell^{2}(\mathbb{N})$ is a compact perturbation of the discrete Laplacian

$$
J_{0}: \quad a_{n}=c_{n}=1, \quad b_{n}=0 .
$$

The structure of the spectrum for such operators is well known: $\sigma(J)=[-2,2] \cup$ $\sigma_{d}(J)$, where $\sigma_{d}(J)$ is at most a denumerable set on the complex plane $\mathbb{C}$ with all accumulation points in $[-2,2]$. We refer to this portion of the spectrum as the discrete spectrum of $J$. The goal of our note is to single out domains on $\mathbb{C}$ free from the discrete spectrum. In particular, a condition on the matrix entries which provides the lack of $\sigma_{d}(J)$ comes in quite naturally. In the case of selfadjoint operators (11) $\left(a_{n}=c_{n}>0, b_{n}=\bar{b}_{n}\right)$ the problem is well elaborated and goes back to M.S. Birman and J. Schwinger (see also [1-3] and the references therein, and [4] [5] for complex Jacobi matrices). The method applied here is totally different and quite elementary. It is adopted from [6] and based on certain lower bounds for

Received by the editors August 18, 2004.

2000 Mathematics Subject Classification. Primary 47B36; Secondary 47 A55.

Key words and phrases. Complex Jacobi matrices, spectrum inclusion regions, Jost function, complex perturbation.

This work was partially supported by INTAS grant no. 03-51-6637. 
the Jost function in the unit disk. The point is made upon the spectrum inclusion regions rather than the bounds for the spectral radius.

\section{RECURRENCE RELATIONS}

We start out with the three-term recurrence relation associated with the matrix $J$ :

(2) $a_{m-1} y_{m-1}+b_{m} y_{m}+c_{m} y_{m+1}=\left(z+z^{-1}\right) y_{m}, \quad m \in \mathbb{N}, \quad z \in \overline{\mathbb{D}}:=\{|z| \leq 1\}$,

$z \neq 0$ (we put $a_{0}=c_{0}=1$ ). It is clear that the initial data $\left\{y_{0}, y_{1}\right\}$ enables one to restore the whole solution $\left\{y_{m}(z)\right\}_{m \geq 0}$ of (2); that is, the dimension of the space of solutions is 2 . Sometimes it is beneficial to deal with a slightly modified relation. If we multiply (2) by $k(j)=\prod_{i=j}^{\infty} a_{i}$ (the product will always be assumed to converge) and put $x_{m}=k(m) y_{m}$, we come to

$$
x_{m-1}+b_{m} x_{m}+a_{m} c_{m} x_{m+1}=\left(z+z^{-1}\right) x_{m}, \quad m \in \mathbb{N} .
$$

\section{FROM RECURRENCE RELATIONS TO DISCRETE INTEGRAL EQUATIONS}

The key role in what follows is played by certain solutions of (2), (3) that have a specific behavior at infinity. We show that such solutions exist as long as the coefficients in (2) tend to their limits fast enough.

Denote by $G$ the Green kernel

$$
G(n, m ; z)=\left\{\begin{array}{cl}
\frac{z^{m-n}-z^{n-m}}{z-z^{-1}}, & m>n, \\
0, & m \leq n,
\end{array} \quad n, m \in \mathbb{Z}_{+}:=\{0,1, \ldots\}, \quad z \neq 0 .\right.
$$

It is clear that

$$
G(n, m, z)=U_{m-n-1}\left(\frac{z+z^{-1}}{2}\right),
$$

where $U_{k}$ is the Chebyshev polynomial of the second kind. The recurrence relations for $G$ are straightforward:

$$
\begin{aligned}
& G(n, m+1 ; z)+G(n, m-1 ; z)-\left(z+z^{-1}\right) G(n, m ; z)=\delta(n, m), \\
& G(n-1, m ; z)+G(n+1, m ; z)-\left(z+z^{-1}\right) G(n, m ; z)=\delta(n, m),
\end{aligned}
$$

where $\delta(n, m)$ is the Kronecker symbol.

We begin with the following conditional result.

Proposition 1. Suppose that equation (3) has a solution $v_{n}$ with asymptotic behavior at infinity

$$
\lim _{n \rightarrow \infty} v_{n}(z) z^{-n}=1
$$

for some $z \in \mathbb{D}$. Then $v_{n}$ satisfies a discrete integral equation

$$
v_{n}(z)=z^{n}+\sum_{m=n+1}^{\infty} J(n, m ; z) v_{m}(z), \quad n \in \mathbb{Z}_{+}
$$

with

$$
J(n, m ; z)=-b_{m} G(n, m ; z)+\left(1-a_{m-1} c_{m-1}\right) G(n, m-1 ; z) .
$$


Proof. Let us multiply (15) by $v_{m}$, (3) by $G(n, m)$ and subtract the latter from the former:

$$
\begin{gathered}
G(n, m+1) v_{m}+G(n, m-1) v_{m}-G(n, m) v_{m-1}-b_{m} G(n, m) v_{m}-a_{m} c_{m} G(n, m) v_{m+1} \\
=\delta(n, m) v_{m} .
\end{gathered}
$$

Summing up over $m$ from $n$ to $N$ gives

$$
\begin{gathered}
v_{n}=\sum_{m=n}^{N}\left\{-b_{m} G(n, m)+\left(1-a_{m-1} c_{m-1}\right) G(n, m-1)\right\} v_{m} \\
+G(n, N+1) v_{N}-a_{N} c_{N} G(n, N) v_{N+1} .
\end{gathered}
$$

For $|z|<1$ we have by (4) and (7),

$$
\lim _{N \rightarrow \infty}\left(G(n, N+1) v_{N}-a_{n} c_{n} G(n, N) v_{N+1}\right)=z^{n},
$$

which along with $J(n, n)=0$ leads to (8), as needed.

The converse statement is equally simple.

Proposition 2. Each solution $\left\{v_{n}(z)\right\}_{n \geq 0}, z \in \overline{\mathbb{D}}$, of equation (8) satisfies the three-term recurrence relation (3).

Proof. Write, for $n \geq 1$,

$$
\begin{aligned}
v_{n-1}+v_{n+1} & =z^{n-1}+z^{n+1}+\sum_{m=n}^{\infty} J(n-1, m) v_{m}+\sum_{m=n+2}^{\infty} J(n+1, m) v_{m} \\
& =\left(z+z^{-1}\right) z^{n}+J(n-1, n) v_{n}+J(n-1, n+1) v_{n+1} \\
& +\sum_{m=n+2}^{\infty}\{J(n-1, m)+J(n+1, m)\} v_{m} .
\end{aligned}
$$

By (44), (9) and (6),

$$
J(n-1, n ; z)=-b_{n}, \quad J(n-1, n+1 ; z)=-\left(z+z^{-1}\right) b_{n+1}+1-a_{n} c_{n}
$$

and

$$
J(n-1, m ; z)+J(n+1, m ; z)=\left(z+z^{-1}\right) J(n, m ; z) .
$$

Hence

$$
\begin{aligned}
v_{n-1}+v_{n+1}+b_{n} v_{n}-\left(1-a_{n} c_{n}\right) v_{n+1} & =\left(z+z^{-1}\right)\left(z^{n}+\sum_{m=n+1}^{\infty} J(n, m) v_{m}\right) \\
& =\left(z+z^{-1}\right) v_{n},
\end{aligned}
$$

which is exactly (3).

\section{The Jost Solution}

To analyze equation (8) it seems reasonable to introduce new variables:

$$
\tilde{v}_{n}(z):=v_{n} z^{-n}, \quad \tilde{J}(n, m ; z):=J(n, m ; z) z^{m-n},
$$

so that

$$
\tilde{v}_{n}(z)=1+\sum_{m=n+1}^{\infty} \tilde{J}(n, m ; z) \tilde{v}_{m}(z), \quad n \in \mathbb{Z}_{+} .
$$


Now $\tilde{J}(n, m ; \cdot)$ is a polynomial and since

$$
\left|G(n, m, z) z^{m-n}\right|=\frac{\left|z^{2(m-n)}-1\right|}{\left|z-z^{-1}\right|} \leq|z| \min \left\{|m-n|, \frac{2}{\left|z^{2}-1\right|}\right\},
$$

the kernel $\tilde{J}$ is bounded by

$$
\begin{aligned}
|\tilde{J}(n, m ; z)| & \leq|z| d_{m} \min \left\{|m-n|, \frac{2}{\left|z^{2}-1\right|}\right\}, \\
d_{m} & :=\left|b_{m}\right|+\left|1-a_{m-1} c_{m-1}\right|, \quad z \in \overline{\mathbb{D}} .
\end{aligned}
$$

The main result concerning equation (8) is the following.

Theorem 1. (i) Suppose that

$$
\sum_{m=1}^{\infty} d_{m}<\infty
$$

Then equation (8) has a unique solution $v_{n}$ such that $v_{n}$ is analytic in $\mathbb{D}$, continuous on $\mathbb{D}_{1}:=\overline{\mathbb{D}} \backslash\{ \pm 1\}$ and 1

$$
\left|v_{n}-z^{n}\right| \leq|z|^{n}\left\{\frac{2|z|}{\left|z^{2}-1\right|} \sum_{m=n+1}^{\infty} d_{m}\right\} \exp \left\{\frac{2|z|}{\left|z^{2}-1\right|} \sum_{m=n+1}^{\infty} d_{m}\right\}, \quad z \in \mathbb{D}_{1}, n \in \mathbb{Z}_{+} .
$$

(ii) Suppose that

$$
\sum_{m=1}^{\infty} m d_{m}<\infty
$$

Then $v_{n}$ is analytic in $\mathbb{D}$, continuous on $\overline{\mathbb{D}}$ and

$$
\left|v_{n}-z^{n}\right| \leq|z|^{n}\left\{\sum_{m=n+1}^{\infty} m d_{m}\right\} \exp \left\{\sum_{m=n+1}^{\infty} m d_{m}\right\}, \quad z \in \mathbb{D}, \quad n \in \mathbb{Z}_{+} .
$$

Proof. The method of successive approximations does the job. Write (10) as

$$
f_{n}(z)=g_{n}(z)+\sum_{m=n+1}^{\infty} \tilde{J}(n, m ; z) f_{m}(z)
$$

with

$$
f_{m}(z):=\tilde{v}_{m}(z)-1, \quad g_{n}(z):=\sum_{m=n+1}^{\infty} \tilde{J}(n, m ; z) .
$$

(i) Put $\sigma_{0}(n):=\sum_{m=n+1}^{\infty} d_{m}, \phi(z):=2|z|\left|z^{2}-1\right|^{-1}$ and apply (11) in the form

$$
|\tilde{J}(n, m ; z)| \leq \phi(z) d_{m}, \quad z \in \mathbb{D}_{1} .
$$

Then the series in (17) converges uniformly on compact subsets of $\mathbb{D}_{1}$ and so $g_{n}$ is analytic in $\mathbb{D}$ and continuous on $\mathbb{D}_{1}$. Let us begin with $f_{n, 1}=g_{n}$ and denote

$$
f_{n, j+1}(z):=\sum_{m=n+1}^{\infty} \tilde{J}(n, m ; z) f_{m, j}(z) .
$$

\footnotetext{
${ }^{1}$ Following the terminology of the selfadjoint case, we call this solution the Jost solution. The function $v_{0}$ is known as the Jost function.
} 
We prove by induction starting with $j=1$ that

$$
\left|f_{n, j}(z)\right| \leq \frac{\left(\phi(z) \sigma_{0}(n)\right)^{j}}{(j-1) !} .
$$

It is obvious for $j=1$ by (18). Next, let (19) be true. Then

$$
\left|f_{n, j+1}(z)\right| \leq \phi(z) \sum_{m=n+1}^{\infty} d_{m}\left|f_{m, j}(z)\right| \leq \frac{(\phi(z))^{j+1}}{(j-1) !} \sum_{m=n+1}^{\infty} d_{m} \sigma_{0}^{j}(m) .
$$

An elementary inequality $(a+b)^{j+1}-a^{j+1} \geq(j+1) b a^{j}$ gives

$$
\sum_{m=n+1}^{\infty} d_{m} \sigma_{0}^{j}(m) \leq \frac{1}{j} \sum_{m=n+1}^{\infty}\left\{\sigma_{0}^{j+1}(m-1)-\sigma_{0}^{j+1}(m)\right\}=\frac{\sigma_{0}^{j+1}(n)}{j},
$$

which proves (19) for $f_{n, j+1}$. Thereby the series

$$
f_{n}(z)=\sum_{j=1}^{\infty} f_{n, j}(z)
$$

converges uniformly on compact subsets of $\mathbb{D}_{1}$ and solves (16), being analytic in $\mathbb{D}$ and continuous on $\mathbb{D}_{1}$. The estimate (13) follows from (19) and $\tilde{v}_{n}=v_{n} z^{-n}$.

Suppose that there are two solutions $f_{n}$ and $\tilde{f}_{n}$ of (16). Take the difference and apply (18):

$$
h_{n} \leq \sum_{m=n+1}^{\infty} \phi(z) h_{m} d_{m}=q_{n}, \quad h_{n}:=\left|f_{n}(z)-\tilde{f}_{n}(z)\right| .
$$

Clearly, $q_{n} \rightarrow 0$ as $n \rightarrow \infty$ and $q_{k}=0$ for some $k$ implies by (20) $h_{n} \equiv 0$. If $q_{n}>0$, then

$$
\frac{q_{n-1}-q_{n}}{q_{n}}=\frac{h_{n} \phi(z) d_{n}}{q_{n}} \leq \phi(z) d_{n}, \quad q_{k} \leq \prod_{j=k+1}^{M}\left(1+\phi(z) d_{j}\right) q_{M},
$$

which leads to $q_{k}=0$ and again $h_{n} \equiv 0$. So the uniqueness is proved.

(ii) The same sort of reasoning is applicable with

$$
|\tilde{J}(n, m ; z)| \leq|z||m-n| d_{m} \leq m d_{m}
$$

and

$$
\left|f_{n, j}(z)\right| \leq \frac{\sigma_{1}^{j}(n)}{(j-1) !}, \quad \sigma_{1}(n):=\sum_{m=n+1}^{\infty} m d_{m}
$$

instead of (18) and (19), respectively.

Corollary 1. Let $t$ be the root of the equation

$$
t e^{t}=1, \quad t \approx 0.567 .
$$

Under assumption (12) the Jost function $v_{0}$ does not vanish in the domain

$$
\Omega:=\left\{z \in \mathbb{D} ; \quad\left|z-z^{-1}\right|>2 t^{-1} \sum_{m=1}^{\infty} d_{m}\right\} .
$$

Under assumption (14) $v_{0}$ does not vanish in $\mathbb{D}$ as long as

$$
\sum_{m=1}^{\infty} m d_{m}<t
$$




\section{Eigenvalues of the JACOBI MATRIX AND Zeros of the Jost FunCtion}

Going back to the matrix $J$, let $\lambda \in \sigma_{d}(J)$ with an eigenvector $h=\left\{h_{n}\right\}_{n \geq 1}$. Clearly, $h_{1} \neq 0$. As a result there is only one linearly independent eigenvector corresponding to any eigenvalue $\lambda$ (an appropriate linear combination of two would give zero). Denote by

$$
Z=Z(J)=\left\{z \in \mathbb{D}: v_{0}(z)=0\right\}
$$

the zero set of the Jost function $v_{0}$ in $\mathbb{D}$. As $\left\{v_{n}\right\}_{n \geq 0} \in \ell^{2}$, for each $z_{0} \in Z$ the vector $\left\{v_{n}\left(z_{0}\right)\right\}_{n \geq 1}$ is the eigenvector of $J$ with the eigenvalue $\lambda_{0}=z_{0}+z_{0}^{-1}$. Conversely, let $\left\{g_{n}\right\}$ and $\left\{h_{n}\right\}$ be two solutions of (2). Define their "Wronskian" by

$$
W_{n}(g, h)=g_{n} h_{n+1}-g_{n+1} h_{n}, \quad n \geq 0 .
$$

From (2) it follows that $a_{n-1} W_{n-1}=c_{n} W_{n}$, and iterating gives

$$
W_{n}=\frac{a_{n-1} \ldots a_{0}}{c_{n} c_{n-1} \ldots c_{1}} W_{0} .
$$

Suppose that both solutions are from $\ell^{2}$. Then $W_{n}$ goes to zero as $n \rightarrow \infty$ and since both products in the RHS of (25) converge, $W_{0}=0$. The latter means that $g$ and $h$ are linearly dependent, and so $u_{0}\left(z_{0}\right)=0$. We end up with

\section{Proposition 3.}

$$
\sigma_{d}(J)=\left\{z+z^{-1}: \quad z \in Z(J)\right\} .
$$

Our main result concerning the discrete spectrum of the Jacobi matrices (1) can be displayed as follows.

Theorem 2. Under assumption (12) the domain

$$
G(J)=\left\{z+z^{-1}: \quad z \in \Omega\right\}
$$

with $\Omega$ (23) is free from the discrete spectrum $\sigma_{d}(J)$. The matrix $J$ has no discrete spectrum at all as soon as (24) holds, where $t$ is the solution of (22).

Remark. Suppose that

$$
c=\frac{2}{t} \sum_{m=1}^{\infty} d_{m}<2 .
$$

Then $\sigma_{d}(J)$ is contained in the union of two symmetric rectangles

$$
\sigma_{d}(J) \subset\left\{w: \sqrt{4-c^{2}}<|\operatorname{Re} w|<\sqrt{4+c^{2}}, \quad|\operatorname{Im} w|<\frac{c^{2}}{4}\right\} .
$$

\section{REFERENCES}

[1] Geronimo J.S. An upper bound on the number of eigenvalues of an infinite dimensional Jacobi matrix, J. Math. Phys. v.23, 1982, p.917-921. MR.0659989(84i:47027)

[2] Geronimo J.S. On the spectra of infinite-dimensional Jacobi matrices, J. Approx. Theory v.53, 1988, p.251-265. MR0947431 (90j:47037)

[3] Hundertmark D., Simon B. Lieb-Thirring inequalities for Jacobi matrices, J. Approx. Theory v.118, 2002, p.106-130. MR.1928259(2003h:39016)

[4] Lyantse V.E. Nonselfadjoint discrete Schrödinger operator, DAN SSSR, v.173, no.6, 1967, p.1260-1263. 
[5] Lyantse V.E. Spectrum and resolvent of a non-selfconjugate difference operator, Ukr. Math. J., v.20, no.4, 1968, p.489-503.

[6] Marchenko V. Sturm-Liouville operators and applications, Kiev, Naukova Dumka, 1977. MR0481179(58:1317)

Institute of Low Temperatures, National Academy of Sciences of Ukraine, 61103 KHARKOV, UKRAINE

E-mail address: egorova@ilt.kharkov.ua

Institute of Low Temperature Physics, National Academy of Sciences of Ukraine, 61103 Kharkov, UKRAINE

E-mail address: golinskii@ilt.kharkov.ua 